\title{
Lagrangian formulations to solve free surface incompressible inviscid fluid flows
}

\author{
Sergio Rodolfo Idelsohn ${ }^{a, *, 1}$, Mario Alberto Storti ${ }^{\text {a,1 }}$, Eugenio Oñate ${ }^{\text {b,2 }}$ \\ a Centro Internacional de Métodos Computacionales en Ingeniería, (INTEC, Universidad Nacional del Litoral, CONICET), \\ Güemes 3450, 3000 Santa Fe, Argentina \\ ${ }^{\mathrm{b}}$ Centre Internacional de Mètodes Numèrics en Enginyeria, CIMNE, Universitat Politècnica de Catalunya, \\ Gran Capità s/n, 08034 Barcelona, Spain
}

Received 5 January 2000; received in revised form 30 October 2000

\begin{abstract}
A particle method is presented for the solution of the incompressible inviscid fluid flow equation using a Lagrangian formulation. The interpolated function are those used in "meshless" approximations and the time integration is introduced in a semi-implicit way by a fractional step method. In this manner, both classical stabilization terms used in incompressible Euler equations are unnecessary: numerical diffusion for convective terms are unnecessary due to the Lagrangian formulation, and stabilization of pressure due to the incompressibility constraint for equal order interpolations is eliminated using the fractional step method. (C) 2001 Elsevier Science B.V. All rights reserved.
\end{abstract}

Keywords: Particle method; Lagrange formulation; Incompressible inviscid fluid flows; Meshless

\section{Introduction}

In the last 20 years computer simulation of the incompressible fluid flow equations using an Eulerian formulation has been required to analyze complex geometry and physics problems. However there are still difficulties to analyze problems in which the shape of the interface changes continuously or fluid structure interactions where large deformations should be considered.

More recently, Particle Methods have been used [2,4,6] in which each particle is followed in a Lagrangian manner. Moving interfaces and boundaries can be analyzed by meshless methods much easier than with the finite element method because it is difficult to fit and move a grid continuously. Furthermore, in Lagrangian formulations the convection terms are calculated by the motion without any numerical diffusion.

The first ideas in this way were proposed by Gingold and Monaghan [5] for the treatment of astrophysical hydrodynamic problems with the method called Smooth Particle Hydrodynamics (SPH). This method was later generalized to fluid mechanic problems $[2,4,6]$. Kernel approximations are used in the SPH method to interpolate the unknowns.

Independently, a family of methods called Meshless Methods have been developed as well for structural [1] as for fluid mechanics problems [8-10]. All these methods use the idea of a polynomial interpolant which fits a number of points minimizing the distance between the interpolated function and the

\footnotetext{
${ }^{*}$ Corresponding author. Tel.: +342-455-6673; fax: +342-455-0944.

E-mail address: rnsergio@arcride.edu.ar (S. Rodolfo Idelsohn).

${ }^{1}$ www.cimec.com.ar.

2 www.cimne.upc.es.
} 
value of the unknown point. These ideas were proposed first by Nayroles et al. [7], used with structural mechanics by Belytschko et al. [1] and with fluid mechanics problems by Oñate et al. [8,9] and Taylor et al. [10].

In this paper, we present new ideas and results about how to solve a particle method in fluid mechanics using meshless approximations instead of kernel functions. In this way, a more general formulation is proposed in which any order of approximation may be used for the unknown functions and derivatives.

\section{Governing equations}

The mass and momentum conservation equations may be written in a semi Lagrangian formulations as:

\section{Mass conservation:}

$$
\begin{aligned}
& \frac{\partial \rho}{\partial t}+\nabla \cdot(\rho \mathbf{u})=0, \\
& \underbrace{\frac{\partial \rho}{\partial t}+\rho \nabla \cdot \mathbf{u}}_{\frac{D \rho}{D t}+\nabla \rho \cdot \mathbf{u}}+\rho \nabla \cdot \mathbf{u}=0 .
\end{aligned}
$$

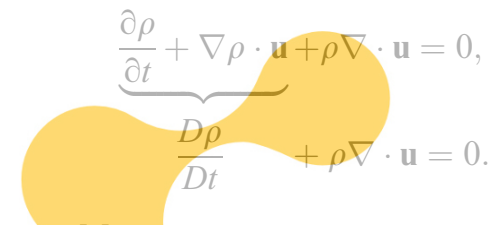

Momentum conservation:
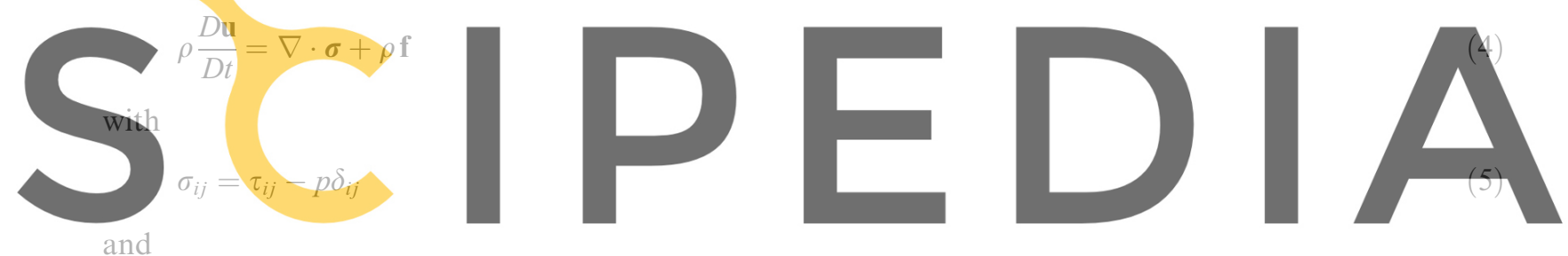

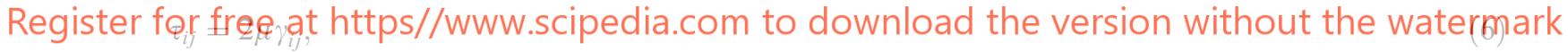

where $\sigma_{i j}$ are the stress tensor, $p$ the pressure, $\mu$ the viscosity, $\gamma_{i j}$ the strain deviatoric tensor and $\mathbf{f}$ a source term.

For non-viscous flow $(\mu \rightarrow 0)$ the 2 set of equations to be used becomes

$$
\begin{aligned}
& \frac{1}{\rho} \frac{D \rho}{D t}=-\nabla \cdot \mathbf{u}, \\
& \frac{D \mathbf{u}}{D t}=-\frac{1}{\rho} \nabla p+\mathbf{f} .
\end{aligned}
$$

\section{The time splitting}

The time integration of Eqs. (7) and (8) presents some difficulties when the fluid is incompressible or near incompressible. In this case, explicit time step can not be used, and even using implicit time step, the incompressibility introduce some wiggles in the pressure solution which must be stabilized. To overcome these difficulties, a fractional step method has been proposed (e.g. [11]) which consist to split each time step in 2 steps as following:

$$
\frac{D \mathbf{u}}{D t} \approx \frac{\mathbf{u}^{n+1}-\mathbf{u}^{n}}{\Delta t}=\frac{\mathbf{u}^{n+1}-\mathbf{u}^{*}+\mathbf{u}^{*}-\mathbf{u}^{n}}{\Delta t}=\frac{\Delta \mathbf{u}^{\prime}+\Delta \mathbf{u}^{*}}{\Delta t}
$$


and

$$
\frac{D \rho}{D t} \approx \frac{\rho^{n+1}-\rho^{n}}{\Delta t}=\frac{\rho^{n+1}-\rho^{*}+\rho^{*}-\rho^{n}}{\Delta t}=\frac{\Delta \rho^{\prime}+\Delta \rho^{*}}{\Delta t},
$$

where $\Delta t=t^{n+1}-t^{n}$ is the time step; $\mathbf{u}^{n}=\mathbf{u}\left(t^{n}, \mathbf{x}^{n}\right), \rho^{n}=\rho\left(t^{n}, \mathbf{x}^{n}\right)$ and $\mathbf{u}^{*}$ and $\rho^{*}$ are fictitious variables defined by the split:

$$
\frac{\Delta \mathbf{u}^{\prime}}{\Delta t}+\frac{\Delta \mathbf{u}^{*}}{\Delta t}=-\frac{1}{\rho} \nabla p+\mathbf{f},
$$

$$
\begin{aligned}
& \frac{\Delta \mathbf{u}^{*}}{\Delta t}=\mathbf{f}, \\
& \frac{\Delta \mathbf{u}^{\prime}}{\Delta t}=-\frac{1}{\rho} \nabla p,
\end{aligned}
$$

$$
\frac{1}{\rho}\left(\frac{\Delta \rho^{\prime}}{\Delta t}+\frac{\Delta \rho^{*}}{\Delta t}\right)=-\nabla \cdot\left[\mathbf{u}^{n+1}-\mathbf{u}^{*}+\mathbf{u}^{*}\right]
$$
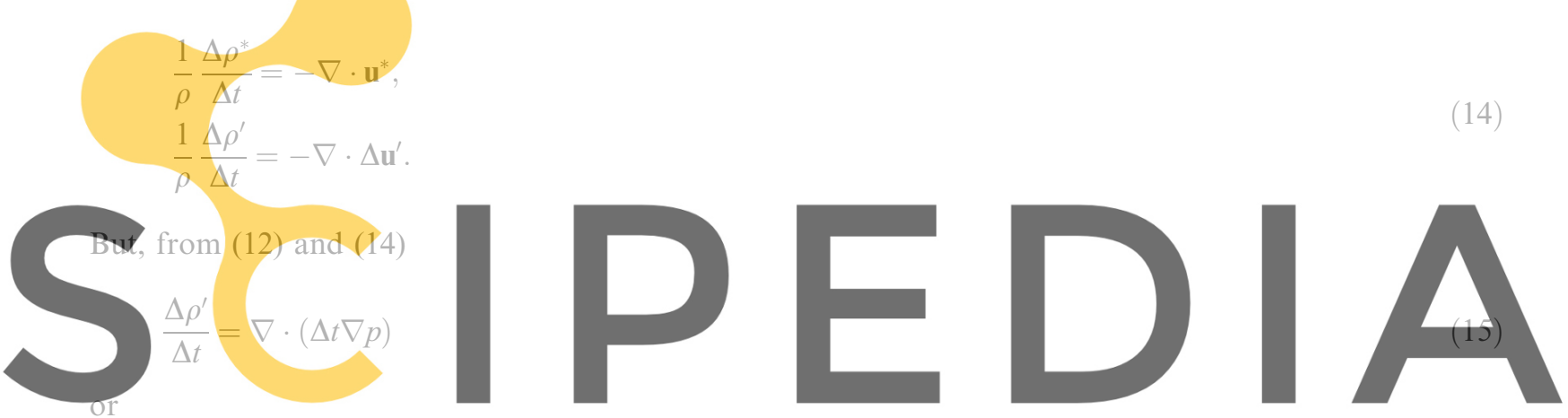

Register for free $e^{*}$ at ktt.tps//www.scipedia.com to download the version without the watermark

where $\nabla^{2}$ is the Laplace operator.

Then, the 6 steps, to achieve a time step are:

(I) From (12) evaluate the $\mathbf{u}^{*}$ velocity.

$$
\mathbf{u}^{*}=\mathbf{u}^{n}+\Delta t \mathbf{f} .
$$

(II) To move the particles

$$
\mathbf{r}^{*}=\mathbf{r}^{n}+\mathbf{u}^{*} \Delta t .
$$

(III) To evaluate the variation of density $\left(\rho^{n+1}-\rho^{*}\right)$.

(IV) To evaluate the pressure solving the Laplacian (15)

$$
\nabla^{2} p=\frac{\rho^{n+1}-\rho^{*}}{\Delta t^{2}}
$$

(V) To evaluate the velocity $\mathbf{u}^{n+1}$ using (12)

$$
\mathbf{u}^{n+1}=\mathbf{u}^{*}-\frac{\Delta t}{\rho} \nabla p .
$$

(VI) To move the particles

$$
\mathbf{r}^{n+1}=\mathbf{r}^{n}+\mathbf{u}^{n+1} \Delta t .
$$




\section{Spatial discretization}

The Lagrangian split scheme described in the previous section has several advantages:

- Step I is linear and explicit. It may be solved at different point positions without any spatial discretization of the velocity. The use of a Lagrangian formulation eliminates the standard convection term present in Eulerian formulations. These convection terms are responsible for non-linearity, non-symmetry and nonself-adjoint operations which need to add high order stabilization terms to avoid numerical oscillations. All these problems are not present in this formulation.

- The split scheme proposed eliminates the standard oscillation appearing in the pressure term when equal order approximations are used near the incompressible flow.

- In all the 6 steps described in Section 3 the only implicit step is the solution of the Laplacian of pressure (step IV), which is a scalar equation, symmetric and positive definite. Then it is very easy to be solved using an iterative scheme.

The big disadvantage of Lagrangian formulation is the permanent updating of the point positions. For this reason, standard finite element method are not useful due to the expensive process of update conforming non-structured finite element meshes. The finite difference method presents difficulties for non-structured meshes, nevertheless, in the last past years, several meshless (non-structured finite difference methods) have been proposed successfully.

In SPH, the unknown function $\phi$ is approximated using kernel functions:

$$
\phi(x)=\int_{\Omega} \phi(\xi) W(\xi) \mathrm{d} \xi \approx \sum_{j=1}^{n} \phi\left(\xi_{j}-\mathbf{x}\right) W\left(\xi_{j}-\mathbf{x}\right) V_{j}=\sum_{j=1}^{n} N_{j} \phi_{j},
$$

where $\Omega$ is a region around the position, $W(\xi)$ is a weighting function, $V_{j}$ an integration weights and

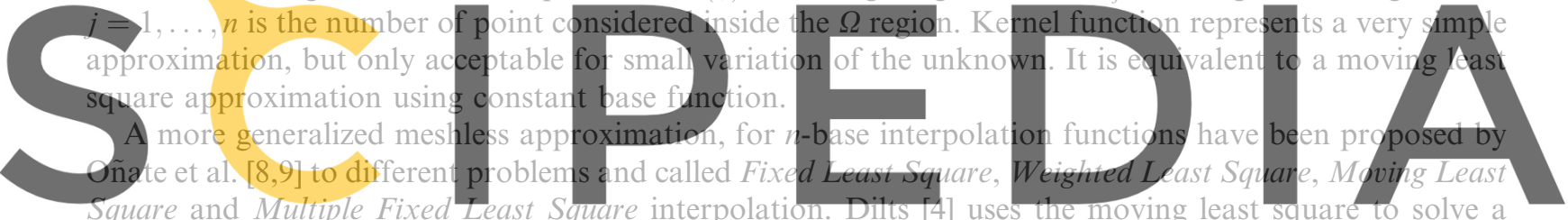

Lagrangian fluid mechanic problem without splitting the time step. Koshizuka and Oka [6] uses the time

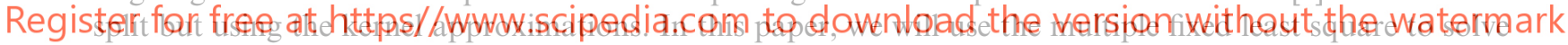

the Laplacian equation obtained from the fractional step method.

\section{Meshless approximation of the function}

An extended version of meshless approximation may be found in Oñate et al. [8,9]. Let $\Omega_{i}$ be the interpolated domain (cloud) of a function $\phi(\mathbf{x})$ and let $s_{j}$, for $j=1, \ldots, n$ a collection of $n$ points with coordinates $\mathbf{x}_{j} \in \Omega_{i}$. The unknown function $\phi$ may be approximated by

$$
\phi(x) \approx \hat{\phi}(x)=\sum_{l=1}^{m} P_{l}(\mathbf{x}) \alpha_{l}=\mathbf{P}(\mathbf{x})^{\mathrm{T}} \boldsymbol{\alpha},
$$

where $P_{l}(\mathbf{x})$ are normally monomial terms and $m$ the number of terms in the approximation, for instance $\mathbf{P}^{\mathrm{T}}(\mathbf{x})=\left[1, x, y, x^{2}, x y, \ldots\right]$.

The function $\hat{\phi}(x)$ can now be sampled at the $n$ points of the cloud giving

$$
\boldsymbol{\phi}^{h}=\left\{\begin{array}{c}
\phi_{1}^{h} \\
\phi_{2}^{h} \\
\vdots \\
\phi_{n}^{h}
\end{array}\right\} \approx\left\{\begin{array}{c}
\hat{\phi}_{1} \\
\hat{\phi}_{2} \\
\vdots \\
\hat{\phi}_{n}
\end{array}\right\}=\left\{\begin{array}{c}
\mathbf{P}_{1}^{\mathrm{T}} \\
\mathbf{P}_{2}^{\mathrm{T}} \\
\vdots \\
\mathbf{P}_{n}^{\mathrm{T}}
\end{array}\right\} \boldsymbol{\alpha}=\mathbf{C} \boldsymbol{\alpha},
$$


where $\phi_{j}^{h}=\phi\left(\mathbf{x}_{j}\right)$ are the unknown values and $\hat{\phi}_{j}=\hat{\phi}\left(\mathbf{x}_{j}\right)$ are the approximate value, and $\mathbf{C}$ is an $n \times m$ matrix. Defining the functional

$$
J=\sum_{j=1}^{n} W\left(\mathbf{x}_{j}\right)\left(\phi_{j}^{h}-\hat{\phi}\left(\mathbf{x}_{j}\right)\right)^{2}
$$

the $\alpha$ parameters may be obtained by minimizing $J$. Then

$$
\begin{aligned}
\boldsymbol{\alpha} & =\mathbf{C}^{-1} \boldsymbol{\phi}^{n}, \quad \mathbf{C}^{-1}=\mathbf{A}^{-1} \mathbf{B}, \\
\mathbf{A} & =\sum_{j=1}^{n} W\left(\mathbf{x}_{j}\right) \mathbf{P}\left(\mathbf{x}_{j}\right) \mathbf{P}^{\mathrm{T}}\left(\mathbf{x}_{j}\right), \\
\mathbf{B} & =\left[W\left(\mathbf{x}_{1}\right) \mathbf{P}\left(\mathbf{x}_{1}\right), W\left(\mathbf{x}_{2}\right) \mathbf{P}\left(\mathbf{x}_{2}\right), \ldots, W\left(\mathbf{x}_{n}\right) \mathbf{P}\left(\mathbf{x}_{n}\right)\right] .
\end{aligned}
$$

Finally, the unknown function remains:

$$
\hat{\phi}(x)=\mathbb{P}^{\mathrm{T}} \mathbf{C}^{-1} \phi^{h}=\sum_{j=1}^{n} N_{j} \phi_{j}^{h}
$$

\section{with the new shape functions $N_{j}$}

$\mathbf{N}=\mathbf{P}^{\mathrm{T}} \mathbf{C}^{-1}$.

This $\mathbf{N}_{j}$ shape functions coincide with the kernel function only for a constant base interpolation
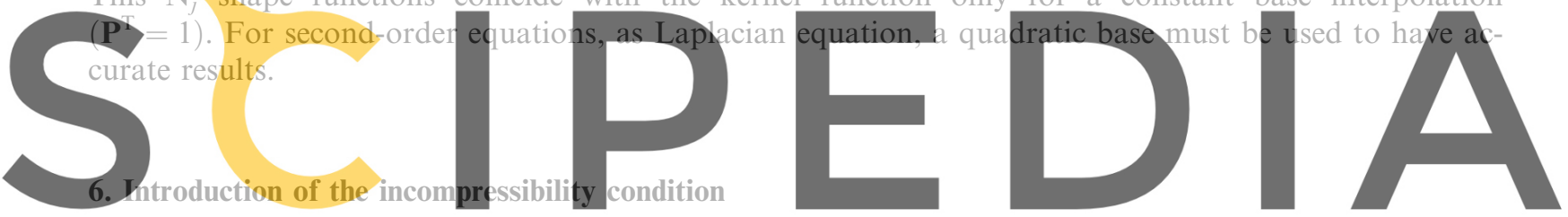

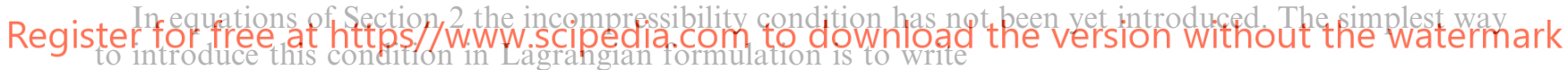

$$
\rho^{n+1}=\rho^{n}=\rho^{0} .
$$

Nevertheless, the $\rho^{*}$ density may be different from $\rho^{0}$.

We will approximate the density using the meshless approximation presented before

$$
\rho(\mathbf{x})=\sum_{j=1}^{n} N_{j} \rho_{j}
$$

The mass conservation implies

$$
\int_{\Omega} \rho \mathrm{d} \Omega=\int_{\Omega}\left(\sum_{j=1}^{n} N_{j} \rho_{j}\right) \mathrm{d} \Omega=\sum_{j=1}^{n} \int_{\Omega} N_{j} \mathrm{~d} \Omega \rho_{j}=\sum_{j=1}^{n} \Omega_{j} \rho_{j}=\text { cnst },
$$

where we have defined the volume $\Omega_{j}$ associated with the particle $j$ by

$$
\Omega_{j}=\int_{\Omega} N_{j} \mathrm{~d} \Omega .
$$

Then, mass conservation implies

$$
\Omega_{i}^{n} \rho_{i}^{n}=\Omega_{i}^{*} \rho_{i}^{*}=\Omega_{i}^{n+1} \rho_{i}^{n+1}=\Omega_{i}^{0} \rho_{i}^{0}
$$


but, introducing the incompressibility condition (31)

$$
\Omega_{i}^{n}=\Omega_{i}^{n+1}=\Omega_{i}^{0}
$$

and then

$$
\rho_{i}^{n+1}-\rho_{i}^{*}=\rho_{i}^{n}\left(\frac{\Omega_{i}^{*}-\Omega_{i}^{0}}{\Omega_{i}^{*}}\right)
$$

which shows that, for the evaluation of the source term of Eq. (19), it is only required the evaluation of the volume $\Omega_{i}^{*}$. The initial volume $\Omega_{i}^{0}$ is constant in time and needs to be evaluated only in the first time step.

It must be also noted that the approximation of the density (Eq. (32)) may be performed with a constant base polynomial ( $m=1$ in Eq. (23)). In this case, the evaluation of the volume $\Omega_{i}^{*}$ is only a sum of the weighting function $W\left(\mathbf{x}_{i}\right)$ in each point of the cloud.

\section{The Laplacian equation}

Once the pressure approximation is defined using the meshless interpolant described in (29), and the source term evaluated with (37), the Laplacian problem (19) may be solved using any of the classical techniques, for instance: point collocation, Galerkin weighted residual method, or other well known minimization techniques. Using a point collocation method, the interpolant base function to approximate the pressure must be second order polynomials. Using some Galerkin weighted residual method, the base functions may be first ord problem in computational inet For this reason, any impro results. The meshless technic matrices needed to solve the Laplacian equation.
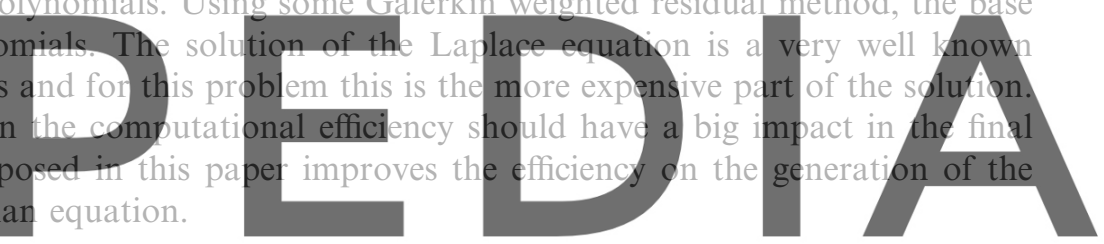

Register for free at https//www.scipedia.com to download the version without the watermark 8. The boundary conditions and free surfaces

Essential or natural boundary condition may be imposed to the solution of the Laplacian Eq. (19). Essential boundary condition may be used on the free surface, where the condition

$$
p=\bar{p}
$$

is imposed ( $\bar{p}$ is the atmospheric pressure). In the other boundaries, the natural boundary condition

$$
\frac{\partial p}{\partial n}=0
$$

where $\hat{\mathbf{n}}$ is the outside normal, may be imposed but, this is not a physical b.c. for the fluid problem. Koshizuka and Oka [6] solved the problem including in the discretization domain, not only the fluid flow, but also a part of the solid container. This solid part must satisfy the same equations, but the velocity remains fixed to the initial value. In this way, in all the boundary domain, including free surface and external solid surface, the essential boundary conditions are imposed.

In order to detect automatically the points which are on the free surface, we also use the algorithm presented by Koshizuka and Oka [6]. A point for which the associated volume $\Omega_{i}^{*}$ satisfies

$$
\Omega_{i}^{*}>\beta \Omega_{i}^{0}
$$

is regarded as a free surface point, where $\beta<1$ is a parameter. 


\section{Numerical solution}

The collapse of a water column was solved by Koshizuka and Oka [6] experimentally and numerically and becomes a classical example to test the validation of Lagrangian formulation in fluid flows. Several authors have solved this problem, (e.g. [3]) but only in the paper of Koshizuka and Oka [6] this problem is integrated in time until the water impinges the right vertical wall and produces a breaking wave.

The water is initially located on the left, supported by a removable board. The collapse starts at time $t=0$, when the removable board is slid up.

The geometry is described in Fig. 1, and the calculation parameters are the following

- Fluid points: 800 ,

- Solid points: 498,

- Initial distance between neighboring points, $l_{0}=0.73 \mathrm{~cm}$,

- Radius of weighting functions $W=2.1 l_{0}$,

- Courant number $C=\Delta t|\mathbf{u}| / l_{0}=0.2$,

- Maximum $\Delta t \leqslant 10^{-3}$,

- Parameter $\beta=0.95$,

- Parameter $\alpha=10^{-6}$.

Figs. 2(a)-(1) shows the point positions at different time steps. The colour scale represents the pressure at the points. Initially, all points are at velocity and pressure zero. During the first time step, the pressure increases near the left corner of the recipient without reaching the hydrostatic pressure which is $28.62 \mathrm{~h} \mathrm{~Pa}$ (Fig. 2(b)). The water is running in the bottom wall until, near $0.3 \mathrm{~s}$, it impinges on the right vertical wall. At this moment pressure rises up very strongly. Due to the incompressibility condition and the infinity rigidity of the wall the pressure reaches, theoretically, an infinite value, which numerically produces an explosion of the particles. In order to avoid this non-physical phenomena a compressible condition is in-

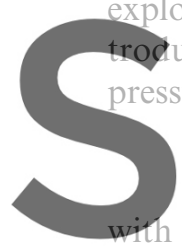

pressure,
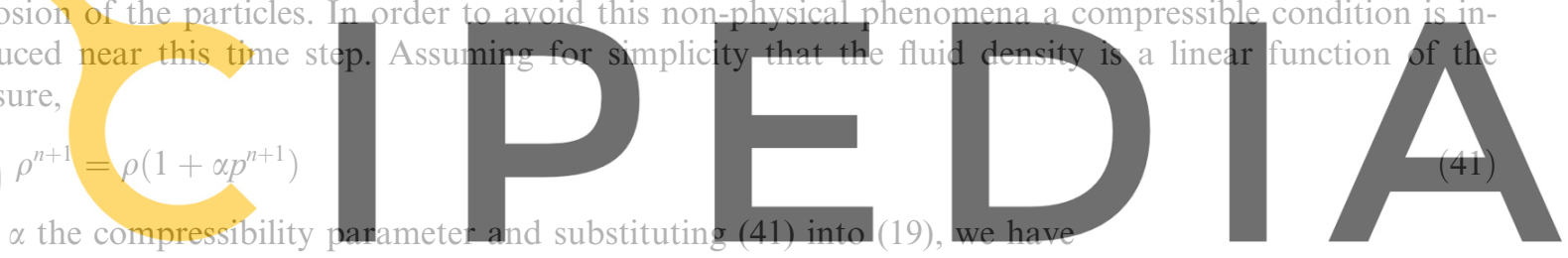

Register for free at https $\Delta t^{2} \nabla^{2} p_{w w}^{n+1}$ w.scipedia.com to download the version without the watermark

Using Eq. (37), the new Laplace operator to be solved becomes

$$
\Delta t^{2} \nabla^{2} p^{n+1}-\rho_{i} \alpha p^{n+1}=\rho_{i}\left(\frac{\Omega_{i}^{*}-\Omega_{i}^{0}}{\Omega_{i}^{*}}\right) .
$$

With this compressibility parameter, the shape of the free surface agrees with the experimental results.

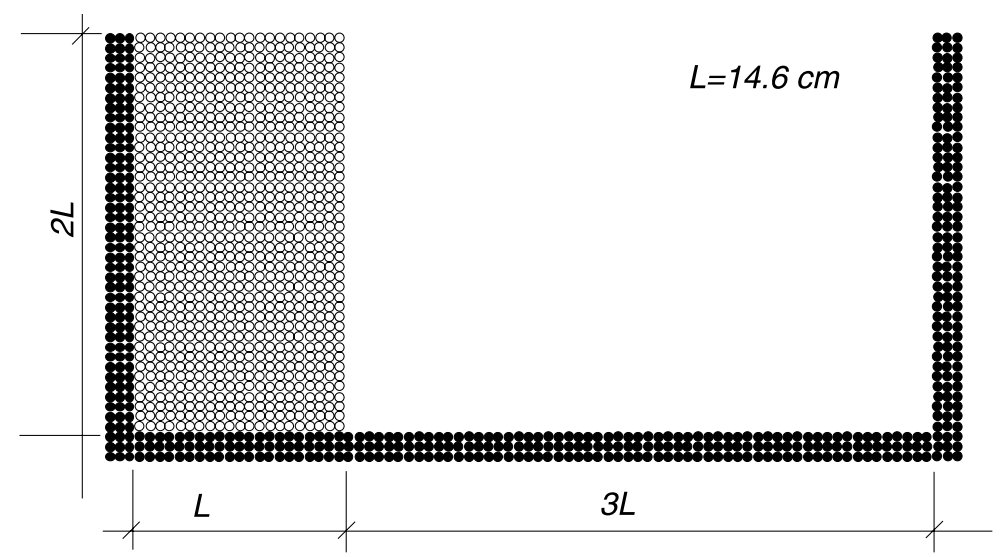

Fig. 1. Water column collapse. Geometry. 


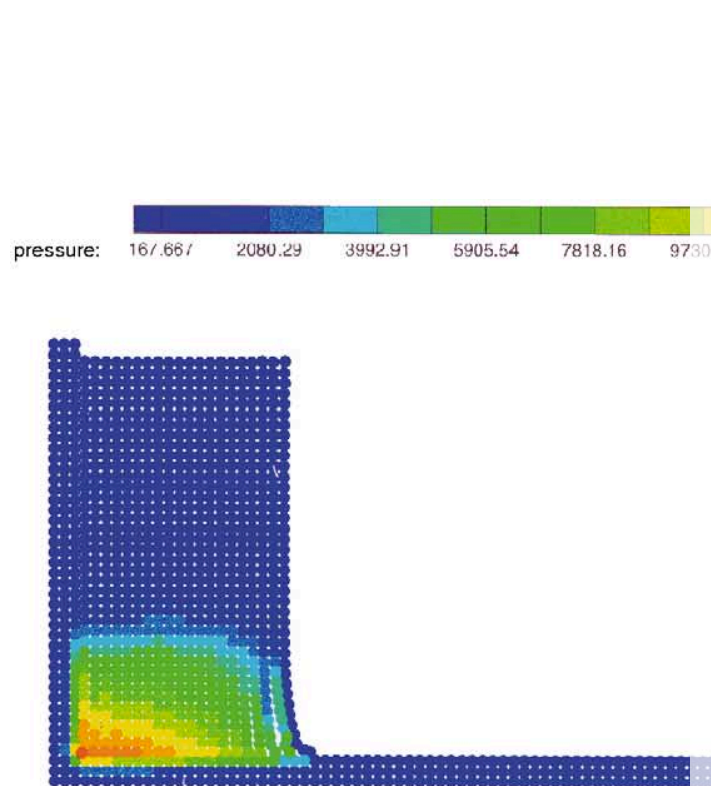

(a)

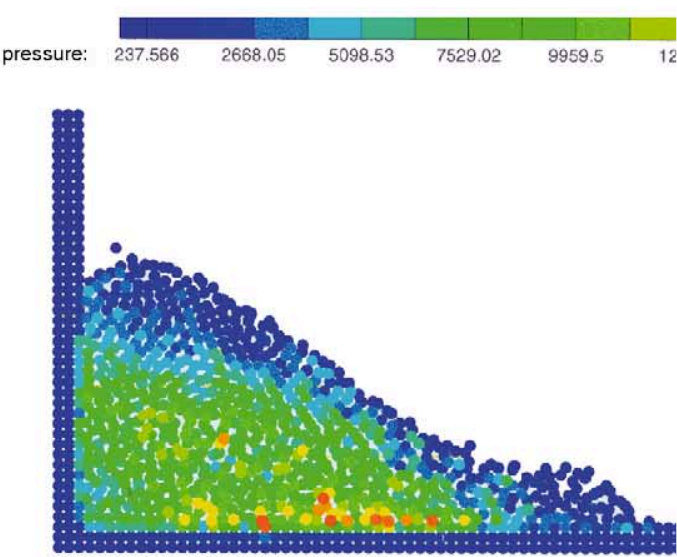

(c)

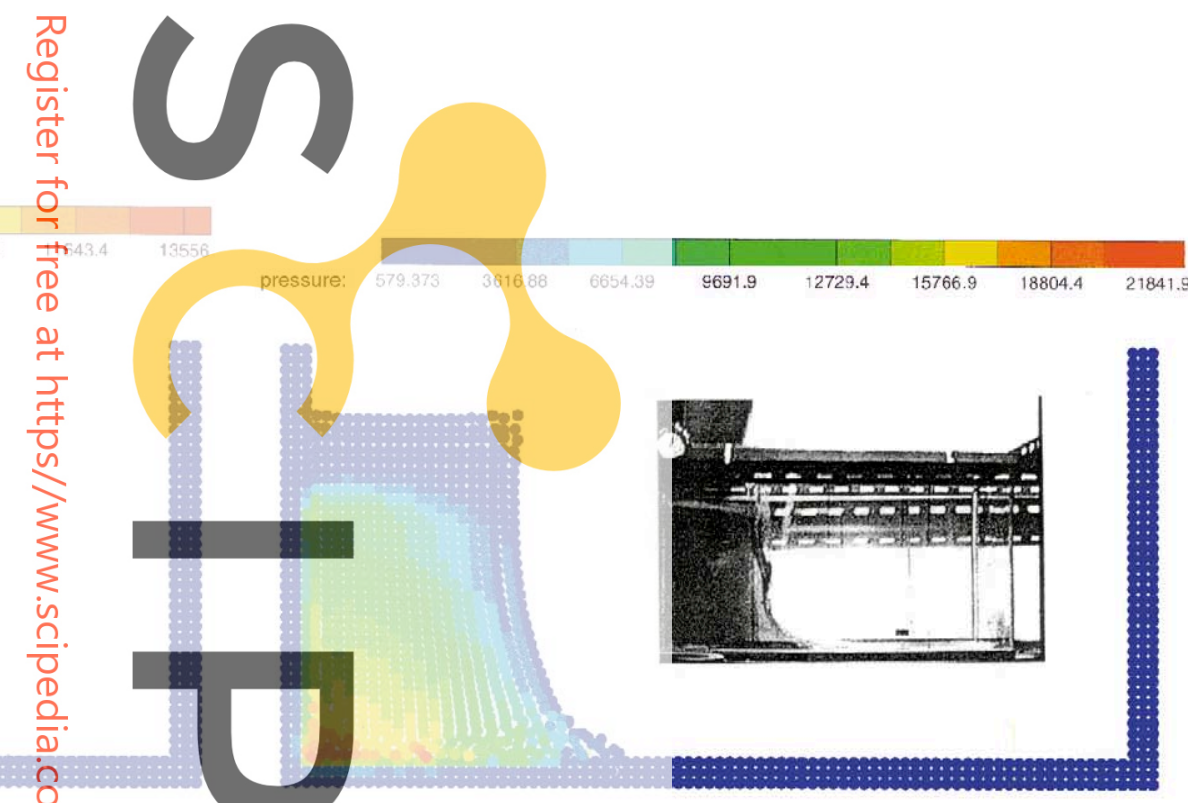

(b)

Fig. 2. Water column collapse. Point positions and pressure values (g) $t=0.50 \mathrm{~s}$; (h) $t=0.60 \mathrm{~s}$; (i) $t=0.70 \mathrm{~s}$; (j) $t=0.80 \mathrm{~s}$; (k) $t=0.90 \mathrm{~s}$;

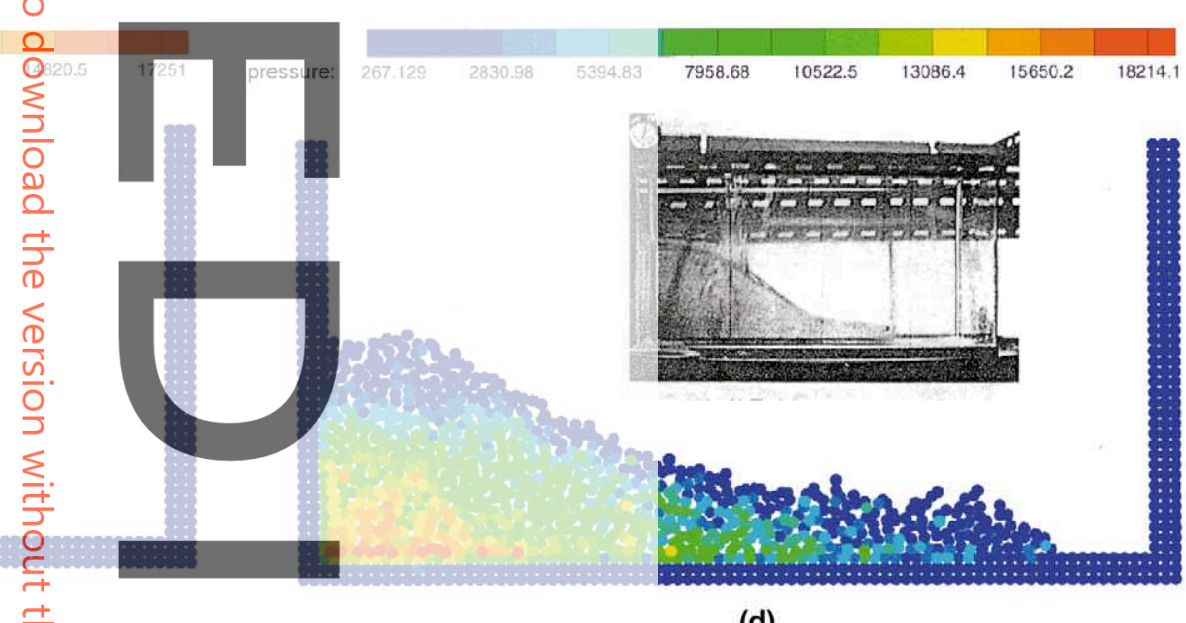

(d)

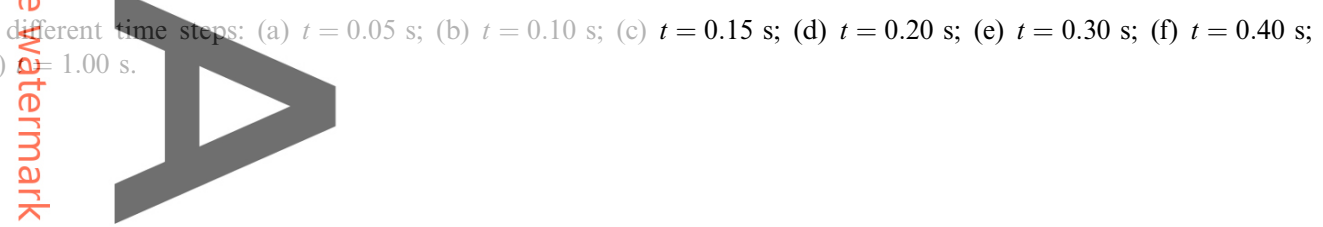




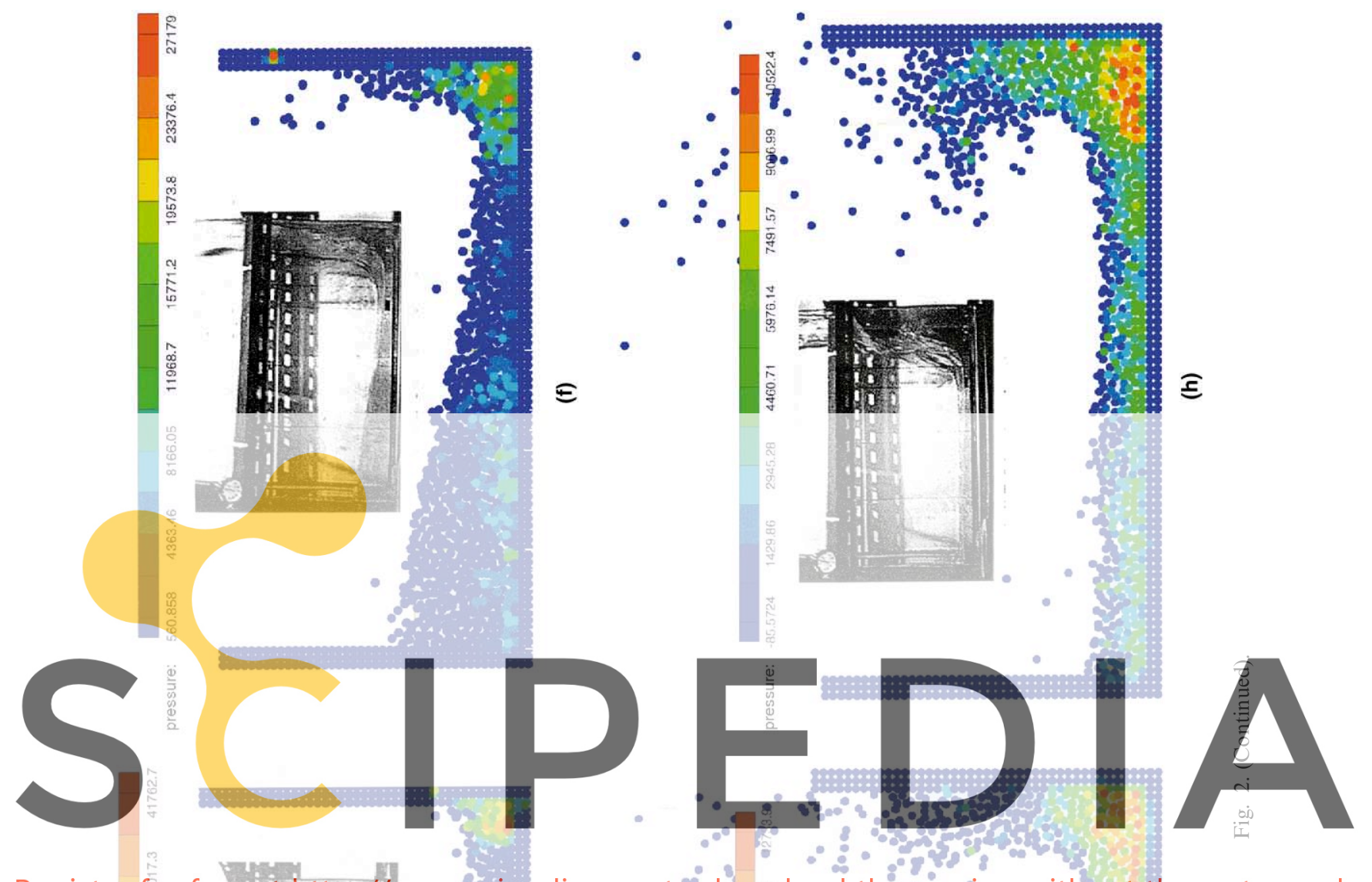

Register for free at https//www.scipedia.com to download the version without the watermark
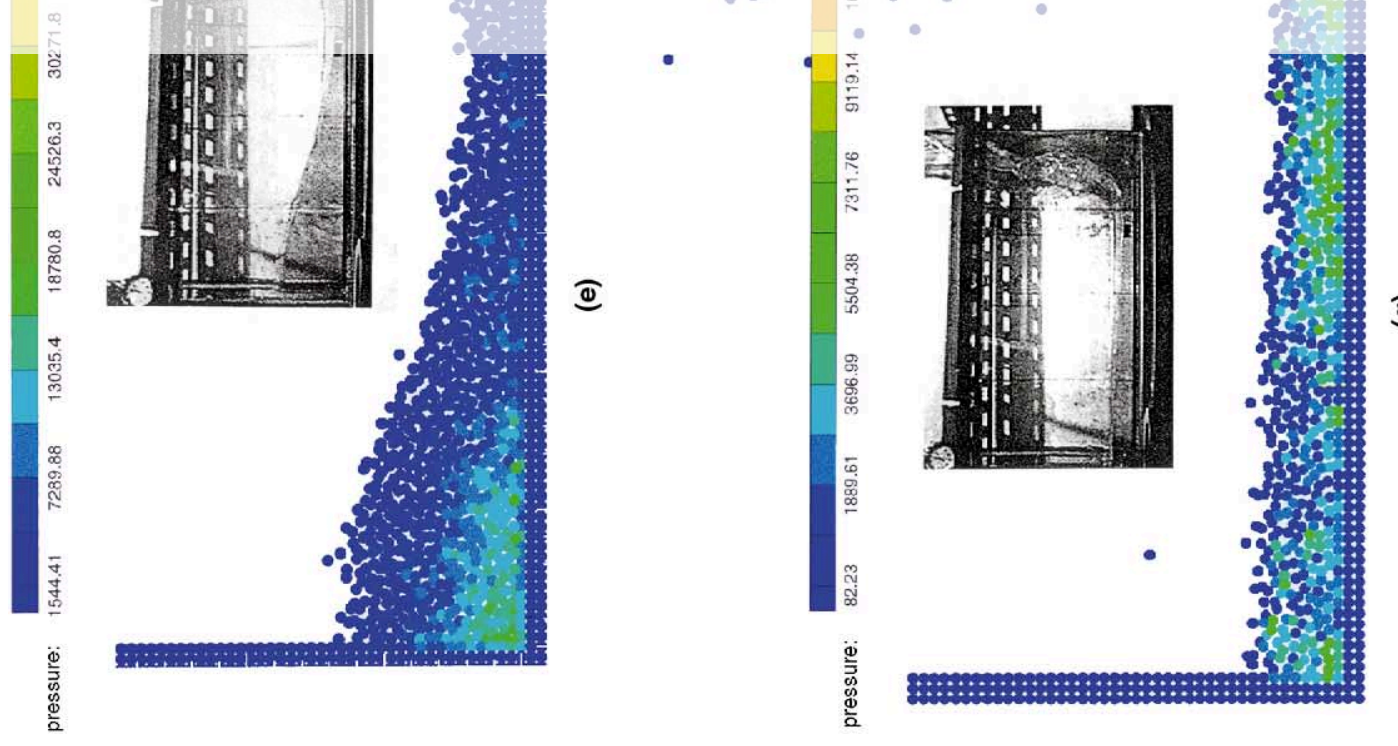

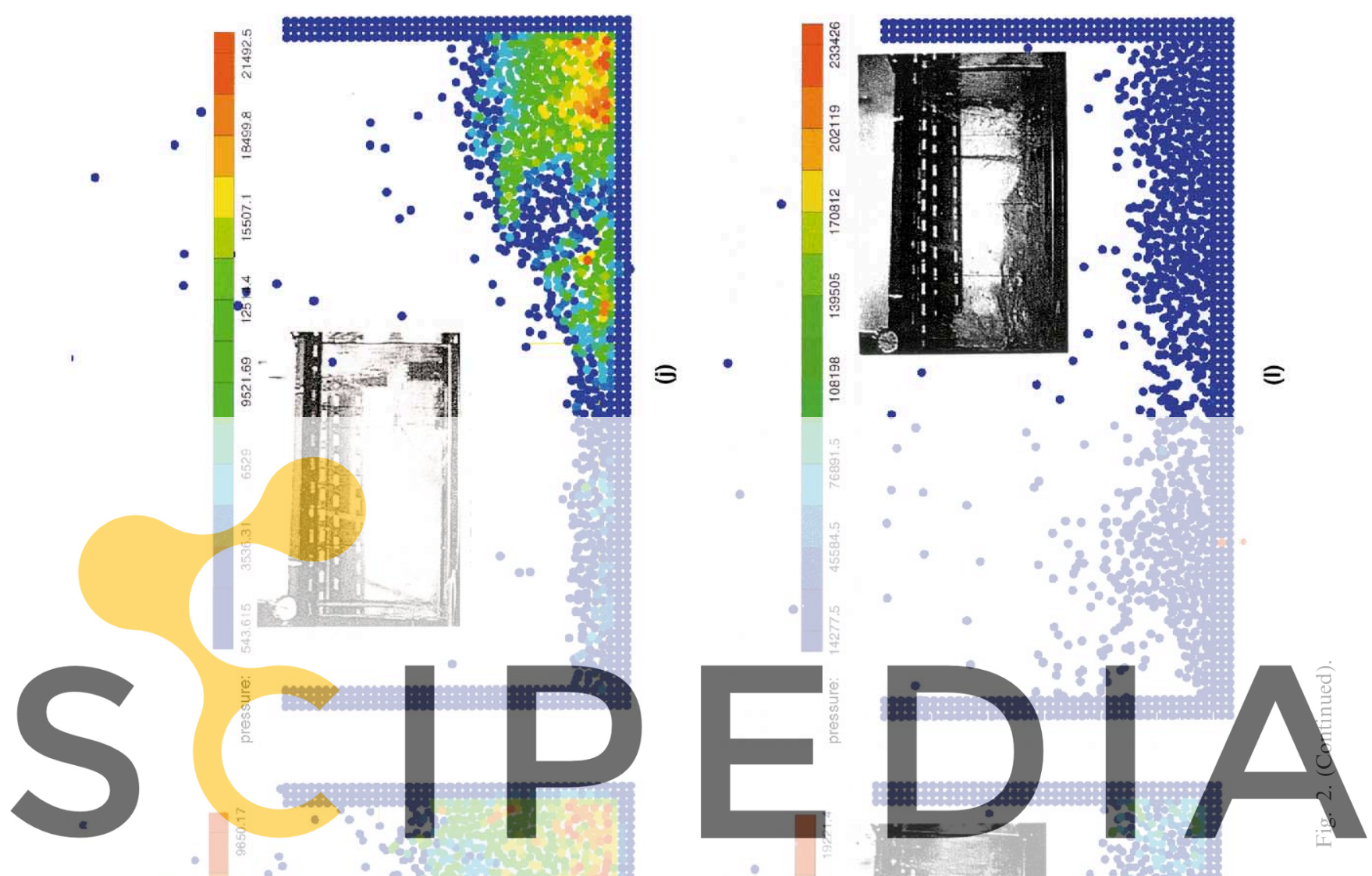

Register for free at https//www.scipedia.com to downloäd the version without the watermark
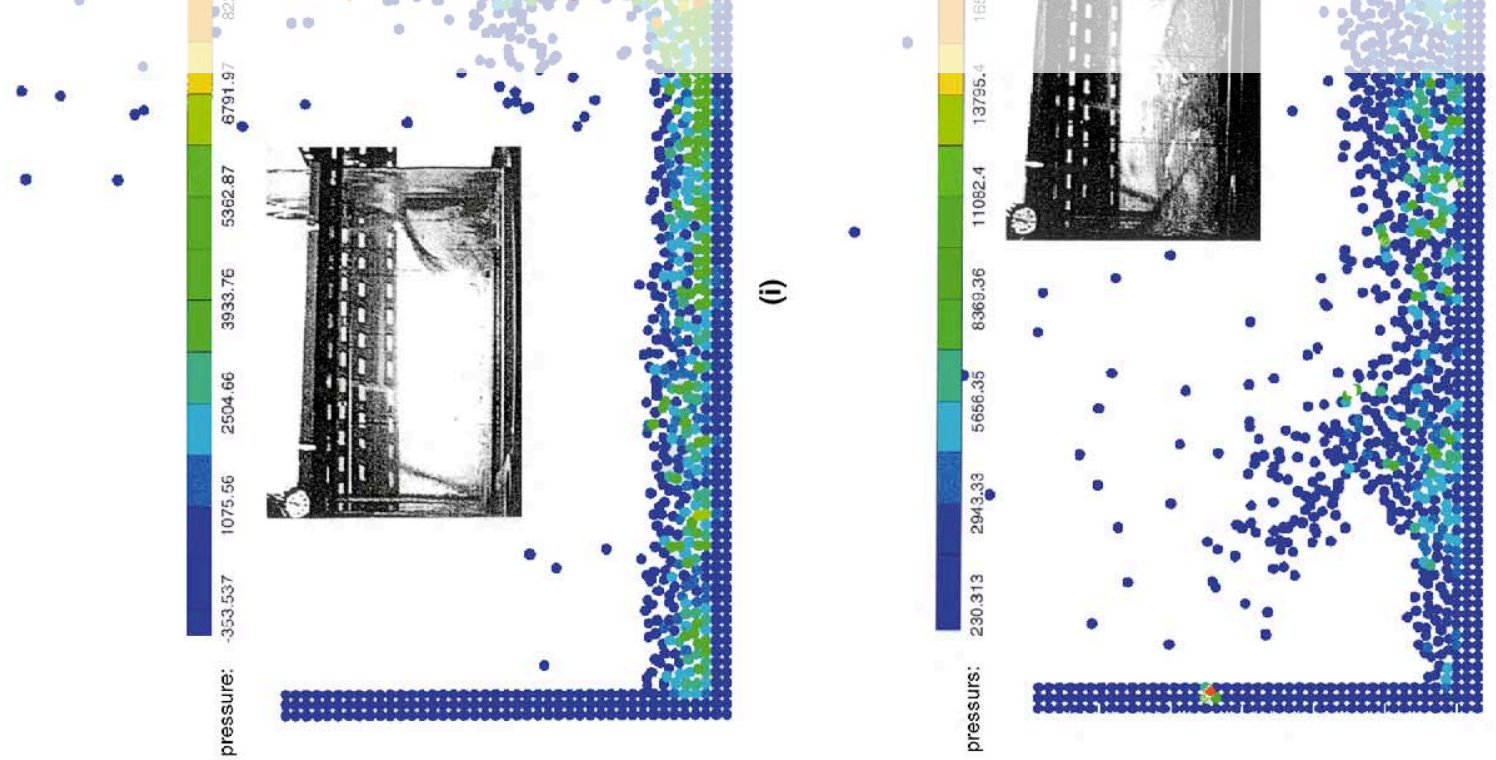
At $t=0.4 \mathrm{~s}$, (Fig. 2(f)) the water goes up losing its momentum, at $0.5 \mathrm{sec}$ it begins to come down. A breaking wave is clear at $t=0.7 \mathrm{~s}$ with a new top on the pressure $p=21,492 \mathrm{~Pa}$ when the wave falls down into the remaining water at $t=0.8 \mathrm{~s}$. A new reflected wave flies toward the left at $0.9 \mathrm{~s}$ reaching the left wall near the first second of the process. Agreement with the experimental results of Koshizuka and Oka [6] are excellent, both in the shape of the free surface as well as in the time developed. The pressure distribution is not described in the experimental results.

\section{Conclusions}

Lagrangian formulations in connection with meshless approximation is an excellent combination to solve fluid mechanical problems, particularly those in which a free surface moves continuously.

Contact problems, as well as breaking waves, may be solved easily without any additional constraint. Furthermore, no stabilization term or evaluation of stabilization parameters are needed in this formulation.

\section{Acknowledgements}

Thanks are given to Mr. Facundo del Pin for his help in preparing the data for the analysis of the collapse of a water column. The first two authors also thank to Consejo Nacional de Investigaciones Científicas y Ténicas (CONICET) and Universidad Nacional del Litoral (UNL).

\section{References}

[1] T. Belytschko, Y. Liu, L. Gu, Element free Galerkin methods, Int. J. Numer. Meth. Eng. 37 (1994) 229-256.

[2] J. Bonet, S. Kulasegaram, Correction and stabilization of smooth particle hydrodynamics methods with applications in metal forming simulation, Int. J. Numer. Meth. Eng., to appear.

[3] J. Bonet, T.S.L. Lok, Variational and momentum preservation aspects of smooth particle hydrodynamics formulations, Comput. Methods Appl. Mech. Eng. 180 (1999) 97-115.

[4] G.A. Dilts, Moving least squares particle hydrodynamics. i. Consistency and stability, Int. J. Numer. Meth. Eng. 44 (1999) 11151155.

[5] R.A. Gingold, J.J. Monaghan, Smoothed particle hydrodynamics theory and application to non-spherical stars, Mon. Natl. Roy. Astr. Soc. 181 (1999) 375-389.

[6] S. Koshizuka, Y. Oka, Moving particle semi-implicit method for fragmentation of incompressible fluid, Nucl. Eng. Sci. 123 (1999) 421-434.

[7] B. Nayroles, G. Touzot, P. Villon, Generalizing the FEM: diffuse approximation and diffuse elements, Comput. Mech. 10 (1992) $307-318$.

[8] E. Oñate, S.R. Idelsohn, O.C. Zienkievicz, R.L. Taylor, A finite point method in computational mechanics, applications to convective transport and fluid flow, Int. J. Numer. Meth. Eng. 39 (22) (1996) 3839-3886.

[9] E. Oñate, S.R. Idelsohn, O.C. Zienkievicz, R.L. Taylor, C. Sacco, A stabilized finite point method for analysis of fluid mechanics problems, Comput. Methods Appl. Mech. Eng. 39 (1996) 315-346.

[10] R.L. Taylor, O.C. Zienkiewicz, E. Oñate, S.R. Idelsohn, Moving least square approximations for solution of differential equations, Internal Report 74, CIMNE, Barcelona, Spain, 1996.

[11] O.C. Zienkiewicz, R. Codina, A general algorithm for compressible and incompressible flow, Part I: The split, characteristic-based scheme, Int. J. Numer. Meth. Fluids 20 (1995) 869-885. 